\title{
Morphine metabolism, transport and brain disposition
}

\author{
Simona De Gregori • Manuela De Gregori • \\ Guglielmina Nadia Ranzani • Massimo Allegri • \\ Cristina Minella $\cdot$ Mario Regazzi
}

Received: 2 September 2011 / Accepted: 2 December 2011 /Published online: 24 December 2011

(C) The Author(s) 2011. This article is published with open access at Springerlink.com

\begin{abstract}
The chemical structures of morphine and its metabolites are closely related to the clinical effects of drugs (analgesia and side-effects) and to their capability to cross the Blood Brain Barrier (BBB). Morphine-6-glucuronide (M6G) and Morphine-3-glucuronide (M3G) are both highly hydrophilic, but only M6G can penetrate the BBB; accordingly, M6G is considered a more attractive analgesic than the parent drug and the M3G. Several hypotheses have been made to explain these differences. In this review we will discuss recent advances in the field, considering brain disposition of M6G, UDPglucoronosyltransferases (UGT) involved in morphine metabolism, UGT interindividual variability and transport proteins.
\end{abstract}

Keywords Blood-Brain-Barrier - Genetic variability ·

Morphine $\cdot$ Morphine-3-glucuronide .

Morphine-6-glucuronide $\cdot$ Transport mechanisms

S. De Gregori $(\bowtie) \cdot$ M. Regazzi

Clinical Pharmacokinetics Unit in Transplantation

and Autoimmune Disease,

Foundation IRCCS Policlinico San Matteo,

P.le Golgi, 2,

7100 Pavia, Italy

e-mail: degregor@unipv.it

M. De Gregori $\cdot$ M. Allegri · C. Minella

Anesthesia Intensive Care and Pain Therapy Service - Fondazione

IRCCS Policlinico San Matteo,

Pavia, Italy

M. De Gregori • G. N. Ranzani

Department of Genetics and Microbiology, University of Pavia, Pavia, Italy

M. Allegri

Department of Surgical Science, Resuscitation-Rehabilitation and

Organ Transplantation, Section of Anaesthesiology,

Resuscitation and Pain Therapy, University of Pavia,

Pavia, Italy

\section{Introduction}

The blood-brain barrier (BBB) is a dynamic interface between the blood and the brain. It eliminates (toxic) substances from the endothelial compartment and supplies the brain with nutrients and other (endogenous) compounds. It can be considered as an organ protecting the brain: its integrity is essential for brain homeostasis, and many carrier-/receptor-mediated transport systems play an important role in maintaining this integrity. Even with minimal disruption of the BBB, circulating neurotoxins, hormones and ions could enter the brain and interfere with the internal milieu, thus resulting in potential damage to neurons (Johansson et al. 1990; Murakami et al. 1997).

Drug transport to the brain depends on various parameters: for drugs that can easily pass the BBB, local blood flow and capillary flow area are limiting factors, whereas for other drugs BBB permeability is restrictive.

Plasma-protein binding controls both the free (unbound) fraction of drugs/compounds and their distribution in the blood; these two factors influence the amount of drugs/ compounds able to pass the BBB. However, it has been observed that many plasma binding proteins (albumin, alpha-acid-glycoprotein, globulins, high-density lipoprotein, low-density lipoprotein) can "donate" ligands to the BBB (De Boer et al. 2003).

Systemic metabolism of drugs takes place mainly in the liver but it can also occur during BBB transport, because many enzymes are present in the brain capillaries (Minn et al. 1991). Before drugs enter the central nervous system (CNS), enzymes can transform them into metabolites that can be more or less effective or even toxic.

The physical-chemical characteristics of drugs (e.g. hydrophilicity, lipophilicity, hydrogen bonding potential) largely determine their passive transport across the $\mathrm{BBB}$, through hydrophilic paracellular and lipophilic transcellular transport. 


\section{UGT genes and proteins}

The main metabolic pathway of morphine in mammals is glucuronidation catalyzed by UDP-glucuronosyltransferase (UGT) enzymes. The human UGTs identified so far have been subdivided into 4 families (UGT1, 2, 3, and 8) according to their sequence homologies (Di et al. 2009). The ability to glucuronidate morphine varies between individuals, and polymorphisms of UGT2B7 have been proposed as a possible cause of this variability (Holthe et al. 2002). Among UGTs, UGT2B7 represents the major isoform responsible for the 3- and 6-glucuronidation of morphine in humans (Fig. 1).

The UGT2 family members (subdivided into UGT2A and $2 \mathrm{~B}$ ) derive from transcription of separate genes located on chromosome $4 q 13$. The UGT2A subfamily includes 3 members, i.e. UGT2A3, which is coded by a single gene of 6 exons, and UGT2A1 and UGT2A2, which share the same $\mathrm{C}$-terminal sequence but have differing $\mathrm{N}$-terminal sequences. UGT2A1 and UGT2A2 proteins arise by the differential splicing of a variable first exon to the same set of 5 downstream exons. UGT2B subfamily includes 7 genes as well as 5 pseudogenes. UGT2B7 variants have been shown to be related to altered drug metabolism and disease risk (Di et al. 2009). For example, a promoter variant of UGT2B7 gene is associated with significantly reduced glucuronidation of morphine in sickle cell disease and contributes to the variability observed in the hepatic clearance of morphine in patients. Innocenti et al. (2008) have recently supported the hypothesis that a common UGT2B7 haplotype (comprised of 10 SNPs) increases enzyme activity by regulating mRNA expression; this might explain part of the phenotypic variability in the pharmacokinetics and pharmacodynamics of UGT2B7 substrates (Innocenti et al. 2008)

\section{Morphine and metabolites}

The major morphine metabolite, morphine-3-glucuronide (M3G), although analgesically inactive, has been reported to antagonize morphine and to produce stimulatory effects, such as myoclonus, seizure and allodynia (Smith 2000). On the other hand, the minor metabolite, morphine-6-glucuronide (M6G), is much more analgesic than morphine.

Morphine is primarily metabolized in the liver by uridine-5'-diphosphate (UDP) glucoronosyltransferase, with specific affinity for the UGT2B7 isoenzyme. This isoenzyme is responsible for the formation of both glucuronide species, but at different amounts (5 times more M3G than M6G). Researchers postulated the presence of another metabolic isoenzyme that largely forms M3G. Although in vitro results have indicated a possible role of UGT1A1 in the formation of $\mathrm{M} 3 \mathrm{G}$, in vivo UGT2B7 isoenzyme remains the primary metabolic enzyme for morphine (Stone et al. 2003). The different formation of $\mathrm{M} 3 \mathrm{G}$ and $\mathrm{M} 6 \mathrm{G}$ metabolites (Fig. 1) is likely due to physicochemical and steric issues that affect the binding of morphine to the phase II enzyme (Coffman et al. 1998).

UGT2B7 is also responsible for the metabolism of several endogenous and exogenous compounds, especially steroid hormones and bilirubin in the newborn. These compounds are competitive substrates for UGT2B7 and can reduce the formation of morphine-conjugate metabolites.
Fig. 1 Morphine-3- and morphine-6-glucuronidation by UGT2B7

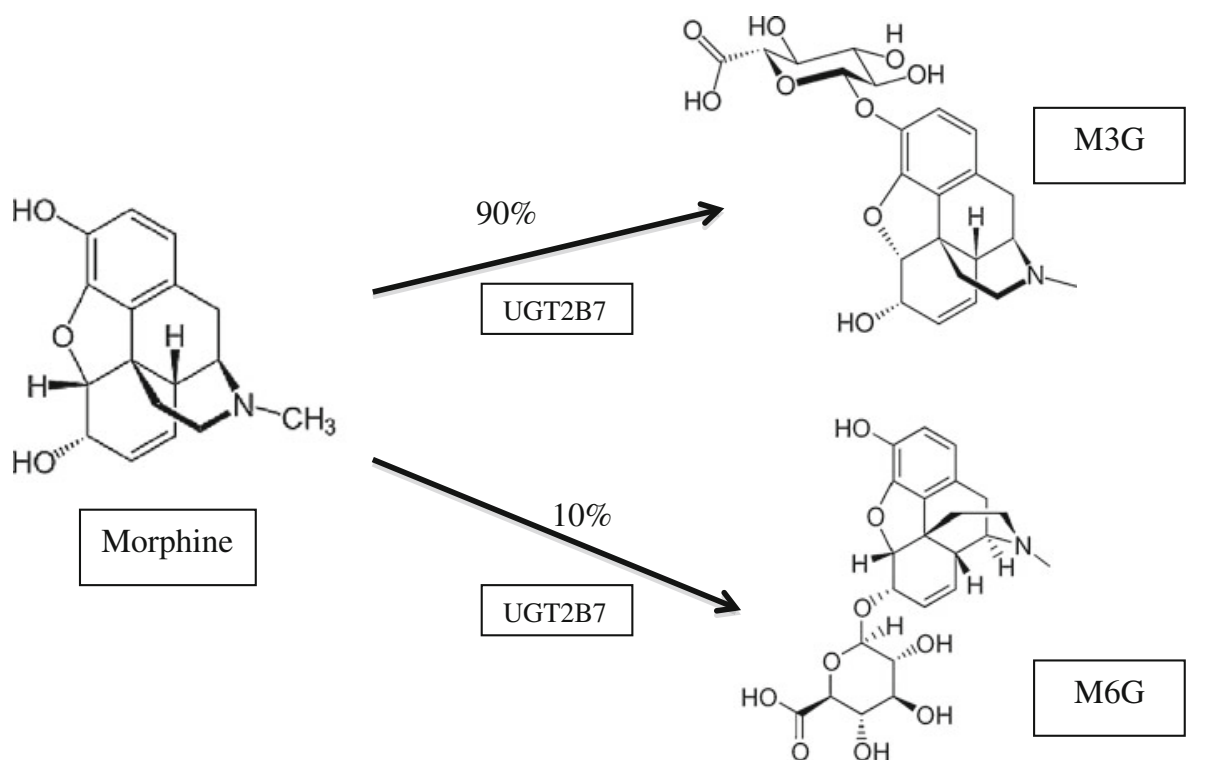


In addition to the liver, human brain homogenates have been shown to metabolize morphine at nanomolar concentrations to $\mathrm{M} 3 \mathrm{G}$ and $\mathrm{M} 6 \mathrm{G}$; therefore, M6G can be formed directly in the CNS and seems to penetrate the BBB at a greater rate than the M6G produced in the liver (Yamada et al. 2003). Interestingly, the M3G/M6G ratio produced by the brain homogenates has been found to be directly associated with morphine concentration. Lower concentrations of morphine corresponded to a lower $\mathrm{M} 3 \mathrm{G} / \mathrm{M} 6 \mathrm{G}$ ratio, perhaps due to the preferential formation of M6G by UGT2B7 (Yamada et al. 2003). Although UGT2B7 appears to play a role in $\mathrm{M} 6 \mathrm{G}$ formation from endogenous morphine, the enzymology of this metabolism in the brain needs to be more thoroughly elucidated. Moreover, the brain UGT isoforms responsible for morphine glucuronidation may be different from the hepatic ones.

Several studies have been performed on morphinemetabolizing enzymes and on the $\mu$-opioid receptor, in order to detect genetic variants possibly contributing to interindividual variability in morphine pharmacology. The UGT2B7 H288Y polymorphism does not seem to account for the significant variations in glucuronide-to-morphine ratio seen in cancer patients (Coughtrie et al. 1989). On the contrary, global UGT activity is apparently modulated by a series of genetic polymorphisms (Coffman et al. 1998). Variants in $\mu$-opioid receptor gene (OPRMI) play an important role in mediating morphine activity: this gene is highly polymorphic, and the rs1799971 SNP in exon 1 seems to be associated with a decreased therapeutic efficacy. In particular, the $118 \mathrm{G}$ homozygotes require higher morphine doses for pain relief than heterozygotes or non-carriers, showing increased risks of intoxication and respiratory depression. The explanation could be related to clinically measurable differences in M6G effects (Lötsch et al. 2002a, b; Lötsch and Geisslinger 2005; Romberg et al. 2005). Similarly, COMT (Catechol-O-MethylTransferase) polymorphisms have to be taken into account for morphine dose modulation: the enzyme is responsible for dopamine, norepinephrine and epinephrine metabolism. Its Val158Met polymorphism (rs4680) is related with enzyme activity since methionine causes a three times reduction of activity, contributing to alterations in pain perception. In particular, patients with Val/Val homozygous genotype require more morphine than Val/Met heterozygous or Met/Met homozygous (reviewed by Allegri et al. 2010).

\section{Morphine glucuronides: Blood Brain Barrier penetration}

In humans, morphine undergoes a variety of metabolic pathways, the most important of which involve the glucuronidation of the 3-OH phenolic group and of the 6$\mathrm{OH}$ alcoholic group to yield morphine $3-\mathrm{O}-\beta$-D-glucuronide (M3G) and morphine 6-O- $\beta$-D-glucuronide (M6G), respectively. Both reactions occur in the liver microsomes; 3 -glucuronidation predominates over 6-glucuronidation for the natural $(-)$-morphine, while the reverse occurs for the unnatural (+)-morphine (Coughtrie et al. 1989).

Following oral administration of morphine to patients, the M6G and M3G plasma AUC (Area Under the concentration-time Curve) exceeds that of the parent drug by factors of 9:1 and 50:1, respectively (Osborne et al. 1990). However, while M3G is not a specific ligand for OPRM1, the receptor shows an affinity for M6G that can be lower than or similar to that of morphine (Christensen and Jǿrgensen 1987). Most of the analgesic effect of morphine treatment is due to the M6G metabolite rather than to morphine itself; accordingly, thanks to its higher potency and less marked respiratory depressive effects, M6G could be a more attractive analgesic compared to the parent drug. The analgesic activity of M6G is extremely potent following intracerebroventricular administration but markedly lower than morphine following systemic administration, due to poor BBB permeability to M6G. Several hypotheses have been advanced to explain this low permeability: one of them relates M6G weak capacity to cross the BBB by passive diffusion to the molecule hydrophilicity. In rats, M6G has been found to be 7.5 times less permeable through the BBB than morphine and similar to sucrose, which is considered as not crossing the BBB (Yoshimura et al. 1973). Another explanation derives from more recent studies suggesting that BBB permeability of M6G is restricted by an efflux mechanism (Bouw et al. 2001). Lötsch et al. (2001) demonstrated, in two strains of mice, the non-influence of Pglycoprotein (P-gp) on M6G pharmacodynamics. This finding was confirmed and further investigated by Bourasset et al. (2003), who co-perfused, in wild-type and P-gp-deficient mice, M6G with digoxin or PSC833, two substrates of Oatp2 transporters; the authors confirmed the absence of M6G transport by P-gp and suggested that M6G is actively transported by a digoxin-sensitive transporter at the BBB level. As Oatp2 is thought to be expressed at both the luminal and basolateral sides of the brain endothelial cells, and to bidirectionally transport its substrates, it can actively transport M6G from blood to brain.

More recent studies (Fujita et al. 2010) in cancer patients have supported the hypothesis that efflux transporter Pglycoprotein (ATP-binding cassette, subfamily B, member 1 (ABCB1)) is a major determinant of the intracellular concentration of morphine and its two main metabolites. ABCB1 pumps the drug out the central nervous system and has a fundamental role in its BBB disposition. Sai et al. (2006) identified a variety of $A B C B 1$ polymorphisms and, more recently, Campa and coworkers (2008) 
demonstrated that pain relief variability in cancer patients is significantly associated with $3435 \mathrm{C}>\mathrm{T}$ variant. On the other hand no relation was found between morphine-induced adverse events and $A B C B 1$ polymorphisms.

In a previous study (Lötsch et al. 2002a, b), PSC833 drug was found to increase threefold the interstitial spinal cord level of M6G, in contrast with its transport decrease across the BBB. These apparently discrepant effects of PSC 833 on M6G may be associated with the different CNS regions analyzed in the two studies: indeed, anatomically and kinetically, there is no reason to expect a parallel behavior of M6G at two distinct CNS barriers, i.e. the BBB and the blood-spinal cord barrier.

M3G, the non-active morphine metabolite, is structurally very similar to $\mathrm{M} 6 \mathrm{G}$, but its transport across the BBB is quite different: for example, probenecid (a substrate for anionic transporters) does not significantly influence M6G pharmacokinetics, but increases the $\mathrm{M} 3 \mathrm{G}$ brain/blood ratio from 0.08 to 0.16 (Xie et al. 2000). An explanation could lie in the different affinity of the two metabolites for the receptors: different from M6G, M3G does not bind $\mu-, \delta$ - and $\mathrm{k}$ opioid receptors (Ulens et al. 2001).

The binding of opioid peptides with $\beta$-D-glucose by an $O-\beta$-linkage has been shown to enhance their brain penetration via the glucose transporter GLUT-1 pathway; accordingly, Polt et al. (1994) suggested that M6G could also cross the BBB by binding the GLUT-1 transporter. This hypothesis was confirmed by Dagenais et al. (2000), who coperfused P-gp competent and deficient mice with a solution of D-glucose (at a concentration high enough to saturate GLUT-1). Irrespective of the mice phenotype, they found that M6G brain uptake was significantly decreased. This finding indicates that M6G can cross the BBB via GLUT1, the same transporter of D-glucose. Like Oatp2, GLUT-1 is expressed on both sides of the brain microvessel endothelial cells and is known to transport glucose by a facilitated passive diffusion pathway. Considering the very low brain uptake, Bourasset et al. (2003) suggested that M6G may be transported from blood to brain and vice versa (according to its concentration gradient) by GLUT-1 and by a digoxin-sensitive transporter (possibly Oatp2) with a weak transport capacity.

Brain penetration can also occur by passive transport, which is difficult to correlate with the two highly polar and hydrophilic morphine conjugates. However, it was observed that the two glucuronides (as zwitterions) exist in a conformational equilibrium between extended and folded forms. The extended conformers, by exposing their polar groups, are highly hydrophilic and predominate in polar media, such as water. In contrast, folded conformers mask part of their polar groups and result more lipophilic in low-polarity media, such as biological membranes (Carrupt et al. 1991). M6G, rather than M3G, prefers the folded form in both neutral and anionic states, because this conformer presents a strong hydrogen bond between the 3-phenolic group and the carboxylic/carboxylate group. The possibility for morphine glucuronides to adapt their polarity to that of the medium could account for their comparatively good brain penetration and slow urinary excretion.

\section{Conclusions}

Morphine is one of the most important and widely used opioid for the treatment of chronic and acute pain: the very wide interindividual variability in the patients' response to the drug may have genetic derivations.

Metabolic pathway (glucoronidation reactions in the liver), sites of action ( $\mu$-opioid receptor) and elimination (efflux from BBB) are regulated by enzymes with an activity that, in many circumstances, is directly related to adverse reactions. It's not yet clear if UGT2B7 genetic polymorphisms-affecting pharmacokinetics-can also cause strong side effects; on the contrary, it was observed that cancer patients homozygous for OPRM1 G allele (A118G) are poor responders to morphine, and require higher doses of the drug to relieve pain. In addition, morphine and its metabolites have to cross the $\mathrm{BBB}$ and penetrate the CNS to perform their activity: it was demonstrated that pain relief variability, in cancer patients, is significantly associated with a $A B C B 1$ gene variant. The current literature clearly indicates that both influx and efflux activities, as well as carrier binding, need to be more thoroughly addressed.

Morphine and its metabolites have a very different ADME profile. The ADME differences are perhaps most likely to explain some of the differences but seem unlikely to be the whole story. We have reviewed the rather scattered literature available on the mechanisms of transport of morphine and its glucuronides (M3GM6G) through the BBB. We believe that a deep understanding of this mechanism, from physical, biochemical and genetic points of view, could improve morphine administration by helping decrease adverse reactions and customize patient pain therapy.

Declaration of interest The authors report no conflicts of interest. The authors alone are responsible for the content

Open Access This article is distributed under the terms of the Creative Commons Attribution Noncommercial License which permits any noncommercial use, distribution, and reproduction in any medium, provided the original author(s) and source are credited. 


\section{References}

Allegri M, De Gregori M, Niebel T et al (2010) Pharmacogenetics and postoperative pain: a new approach to improve acute pain management. Minerva Anestesiol 76(11):937-44

Bourasset F, Cisternino S, Temsamani J, Scherrmann JM (2003) Evidence for an active transport of morphine-6- $\beta$-D-glucuronide but not P-glycoprotein-mediated at the blood-brain barrier. J Neurochem 86:1564-1567

Bouw MR, Tunblad K, Hammarlund-Udenaes M (2001) Blood Brain Barrier transport and brain distribution of morphine-6glucuronide in relation to the antinociceptive effect in rats pharmacokinetic/pharmacodynamic modelling. Br J Pharmacol 134:1796-1804

Campa D, Gioia A, Tomei A et al (2008) Association of ABCB1/ MDR1 and OPRM1 gene polymorphisms with morphine pain relief. Clin Pharmacol Ther 83:559-566

Carrupt PA, Testa B, Bechalany A et al (1991) Morphine 6-Glucuronide and Morphine 3-Glucuronide as molecular chameleons with unexpected lipophilicity. J Med Chem 34:1272-1275

Christensen CB, Jǿrgensen LN (1987) Morphine-6-glucuronide has high affinity for the opioid receptors. Pharmacol Toxicol 60:75-76

Coffman BL, King CD, Rios GR, Tephly TR (1998) The glucuronidation of opioids, other xenobiotics, and androgens by human UGT2B7Y (268) and UGT2B7H(268). Drug Metab Dispos 26(1):73-77

Coughtrie MW, Ask B, Rane A et al (1989) The enantioselective glucuronidation of morphine in rats and humans. Biochem Pharmacol 38:3273-3280

Dagenais C, Rousselle C, Pollack GM, Scherrmann JM (2000) Development of an in situ mouse brain perfusion model and its application to mdr1a P-glycoprotein-deficient mice. J Cereb Blood Flow Metab 20:381-386

De Boer AG, van der Sandt ICJ, Gaillard PJ (2003) The role of drug transporters at the Blood-Brain-Barrier. Annu Rev Pharmacol Toxicol 43:629-656

Di YM, Chan E, Wei MQ et al (2009) Prediction of deleterious nonsynonymous single-nucleotide polymorphisms of human uridine diphosphate glucuronosyltransferase genes. AAPS J 11:469-480

Fujita K, Ando Y, Yamamoto W et al (2010) Association of UGT2B7 and $\mathrm{ABCB} 1$ genotypes with morphine-induced adverse drug reactions in Japanese patients with cancer. Cancer Chemother Pharmacol Jan 65(2):251-8

Holthe M, Klepstad P, Zahlsen K et al (2002) Morphine glucuronideto-morphine plasma ratios are unaffected by the UGT2B7 H268Y and UGT1A1 $* 28$ polymorphisms in cancer patients on chronic morphine therapy. Eur J Clin Pharmacol 58:353-356

Innocenti F, Liu W, Fackenthal D, Ramírez J, Chen P, Ye X, Wu X, Zhang W, Mirkov S, Das S, Cook E Jr, Ratain MJ (2008) Single nucleotide polymorphism discovery and functional assessment of variation in the UDP-glucuronosyltransferase 2B7 gene. Pharmacogenet Genomics 18(8):683-97

Johansson BB, Nordborg C, Westergren I (1990) Neuronal injury after transient opening of the blood-brain barrier: modifying factors. Johansson BB, Owman CG, Widner H. Pathophysiology of the blood-brain barrier. Amsterdam Elsevier 145-157

Lötsch J, Geisslinger G (2005) Are $\mu$-opioid receptor polymorphisms important for clinical opioid therapy? TRENDS Mol Med 11 (2):82-89
Lötsch J, Tegeder I, Angst MS, Geisslinger G (2001) Antinociceptive effects of morphine-6-glucuronide in homozygous MDR1a Pglycoprotein knockout and in wildtype mice in the hotplate test. Life Sci 66:2393-2403

Lötsch J, Schmidt R, Vetter G et al (2002a) Increased CNS uptake and enhanced antinociception of morphine-6-glucuronide in rats after inhibition of P-glycoprotein. J Neurochem 83:241248

Lötsch J, Zimmermann M, Darimont J (2002b) Does the A118G polymorphism at the $\mu$-opioid receptor gene protect against morphine-6-glucuronide toxicity? Anesthesiology 97:814819

Minn A, Ghersi-Egea JF, Perrin R et al (1991) Drug metabolizing enzyme in the brain and cerebral microvessels. Brain Res Rev $16: 65-82$

Murakami K, Kondo T, Chan PH (1997) Blood-brain barrier disruption: edema formation and apoprotic neuronal death following cold injury. Acta Neurochir Suppl (Wien) 70:234-236

Osborne R, Joel S, Trew D, Slevin M (1990) Morphine and metabolite behavior after different routes of morphine administration: demonstration of the importance of the active metabolite morphine-6glucuronide. Clin Pharmacol Ther 47:12

Polt R, Porreca F, Szabo LZ et al (1994) Glycopeptide enkephalin analogues produce analgesia in mice: evidence for penetration of the blood-brain barrier. Proc Natl Acad Sci USA 91:7114-7118

Romberg R, Olofsen E, Bijil H (2005) Polymorphism of mu-opioid receptor gene (OPRM1:c.118A>G) does not protect against opioid induced respiratory depression despite reduced analgesic response. Anesthesiology 102:522-530

Sai K, Itoda M, Saito Y et al (2006) Genetic variations and haplotype structures of the ABCB1 gene in a Japanese population: an expanded haplotype block covering the distal promoter region, andassociated ethnic differences. Ann Hum Genet 70:605-622

Smith MT (2000) Neuroexcitatory effects of morphine and hydromorphone: evidence implicating the 3-glucuronide metabolites. Clin Exp Pharmacol Physiol 27:524-528

Stone A, Mackenzie P, Galetin A et al (2003) Isoform selectivity and kinetics of morphine 3- and 6-glucuronidation by human UDP-glucuronosyltransferases: evidence for atypical glucuronidation kinetics by UGT2B7. Drug Metab Dispos 31:10861089

Ulens C, Baker L, Ratka A et al (2001) Morphine-6betaglucuronide and morphine-3-glucuronide, opioid receptor agonists with different potencies. Biochem Pharmacol 62 (9):1273-1282

Xie R, Bouw M, Hammarlund-Udenaes M (2000) Modelling of the blood-brain barrier transport of morphine-3-glucuronide studied using microdialysis in the rat: involvement of probenecidsensitive transport. Br J Pharmacol 131:1784-1792

Yamada H, Ishii K, Ishii $\mathrm{Y}$ et al (2003) Formation of highly analgesic morphine-6-glucuronide following physiologic concentration of morphine in human brain. J Toxicol Sci 28:395401

Yoshimura H, Ida S, Oguri K, Tsukamoto H (1973) Biochemical basis for analgesic activity of morphine-6-glucuronide. Penetration of morphine-6-glucuronide in the brain of rats. Biochem Pharmacol 22:1423-1430 